\title{
Manuseio de cateter venoso central totalmente implantado: saberes de graduandos de enfermagem
}

\section{Handling of fully implanted central venous catheter: knowledge of nursing students}

\author{
Maria Júlia Oliveira Ramos' $\bullet$ Simone Yuriko Kameo ${ }^{2} \bullet$ Jéssica dos Santos Costa ${ }^{3}$ \\ Bruno Ferreira Amorim ${ }^{4} \bullet$ Guilliana Nunes de Castro Ferreira $^{5} \bullet$ Maria José Oliveira dos Santos $^{6}$ \\ Simone Otília Cabral Neves ${ }^{7} \bullet$ Glebson Moura Silva ${ }^{8}$
}

\begin{abstract}
RESUMO
Objetivo:Analisar os saberes sobre manuseio de cateter venoso central totalmente implantado (CVC-TI) de graduandos em enfermagem da Universidade Federal de Sergipe (UFS). Metodologia:Trata-se estudo exploratório, descritivo, realizado com discentes do segundo ao quinto ciclo do curso de Enfermagem da UFS. Foi realizado um mini curso intitulado "CateterVenoso Central totalmente implantado: procedimentos e cuidados" onde foram aplicados questionários sobre a temática antes e após o mini curso. Resultados:A amostra compreendeu II I discentes; 30,6\% destes responderam corretamente aos questionamentos sobre CVC-TI antes do mini curso ofertado.Após o mini curso, 80,5\% responderam corretamente sobre o tema, com melhora significativa dos conhecimentos adquiridos. Dessa forma, o conhecimento dos graduandos era deficitário antes do mini curso, com melhora significativa após o mesmo. Conclusões: Há necessidade de inserção deste tema nos cursos de graduação em enfermagem com o objetivo de preparar os graduandos para atuação nos serviços de saúde.

Palavras-chave: Enfermagem Oncológica; Cuidados de Enfermagem; Cateterismo Venoso Central;Aprendizagem; Estudantes.
\end{abstract}

\begin{abstract}
Objective:To analyze the knowledge about handling fully implanted central venous catheters of undergraduate nursing students at the Federal University of Sergipe (UFS). Methodology: It is an exploratory descriptive study, carried out with students from the second to the fifth cycle of the Nursing course at UFS. Mini-courses entitled "Central Venous Catheter fully implanted: procedures and care" were carried out, with questionnaires on CVC-TI before and after the mini-course. Results: Sample comprised III students; $30.6 \%$ of these answered correctly the questions about CVC-TI before the short course offered, with greater accuracy in the item handling of CVC-TI.After the short course, $80.5 \%$ answered correctly on the topic, with a significant improvement in the knowledge acquired.The age among the participants in this study was between 17 and 22 years old. The evaluation of knowledge about CVC-TI showed ignorance by nursing students before the mini-course for $70.4 \%$ of the sample. The highest number of correct answers in the questions after participating in the short course on CVC-TI.The knowledge of the students was deficient before the mini-course, with significant improvement after the same. Conclusions: There is a need to include this topic in undergraduate nursing courses in order to prepare undergraduate students to work in health services.
\end{abstract}

Keywords: Oncology Nursing; Nursing Care; Catheterization, Central Venous; Learning; Students.

I Graduanda em Enfermagem pela Universidade Federal de Sergipe (UFS), Campus Lagarto.

2 Professora do Departamento de Educação em Saúde, Universidade Federal de Sergipe, Campus Lagarto, Brasil. Enfermeira pela Universidade Estadual de Londrina. Especialização em Enfermagem Oncológica pelo INCA. Doutorado em Enfermagem pela Escola de Enfermagem de Ribeirão Preto-USP.

3 Enfermeira pela Universidade Federal de Sergipe (UFS) - Campus Lagarto. Residente do Programa de Saúde da Família do Instituto de Medicina Integral Professor Fernando Figueira-IMIP/PE.

4 Graduando em Medicina pela Universidade Federal de Sergipe (UFS), Campus Lagarto.

5 Enfermeira pela Universidade Federal de Sergipe (UFS), Campus Lagarto. Pós-graduanda em Enfermagem Neonatal e Pediátrica pela Faculdade Estácio.

6 Enfermeira pela Universidade Federal de Sergipe (UFS) - Campus Lagarto. Pós-graduada em Enfermagem de Urgência e Emergência pela Faculdade Venda Nova do Imigrante (FAVENI).

7 Professora do Departamento de Educação em Saúde, Universidade Federal de Sergipe, Campus Lagarto, Brasil. Enfermeira pela Universidade Federal de Sergipe (UFS). Mestre em Saúde e Ambiente pela Universidade Tiradentes (UNIT).

8 Professor do Departamento de Enfermagem, Universidade Federal de Sergipe, Campus Lagarto, Brasil. Doutor em Educação pela Universidade Federal de Sergipe. Mestre em Saúde e Ambiente pela Universidade Tiradentes. Especialista em Saúde da Família e Gerenciamento pela Universidade Federal da Bahia. Enfermeiro pela Universidade Estadual do Sudoeste da Bahia. 


\section{INTRODUÇÃO}

O câncer, uma doença crônico-degenerativa, é uma das principais causas de mortes tanto no Brasil quanto no mundo(l).Diante dos avanços tecnológicos, a base do tratamento oncológico é a quimioterapia, a radioterapia e a cirurgia, que será escolhido de acordo com o quadro clínico do paciente e o desenvolvimento da doença( ${ }^{(2)}$.

Dessas vertentes, o tratamento mais utilizado atualmente é a quimioterapia por ter uma propriedade antitumoral sistêmica, permitindo o tratamento precoce de metástases não detectáveis, o que possibilita a cura de vários tipos de câncer ${ }^{(3)}$.

A quimioterapia é um tratamento longo e duradouro, pois exige uso constante da rede venosa do paciente que poderá em algum momento da terapia apresentar fragilidade vascular periférica. Nesses casos, é necessário escoIher outro método que assegure tal tratamento, podendo ser por meio do Cateter Venoso Central Totalmente Implantado (CVC-TI), que vem sendo usado desde $1982^{(3,4)}$.

Nos últimos anos, a Organização Mundial da Saúde (OMS) junto à Joint Commisssion International (JCl) preconizaram a implementação das Metas Internacionais de Segurança do Paciente, destacando-se aquela que visa prevenir as infecções relacionadas à assistência à saúde - IRAS ${ }^{(5,6)}$-, meta básica e de extrema importância ao paciente oncológico que possui o dispositivo.

Por serem pacientes vulneráveis, necessitam de grande atenção da equipe de enfermagem. Essa adoção de medidas visa reduzir a um mínimo aceitável o risco da ocorrência de infecção no cateter decorrente do cuidado em saúde ${ }^{(5)}$.

Segundo a Lei 7.498 do Exercício Profissional de Enfermagem, o enfermeiro é responsável pela prevenção e pelo controle das IRAS ${ }^{(7)}$. Este profissional tem um importante papel nos cuidados com o CVC-TI, sendo o mesmo responsável por cuidados diretos com a manutenção e a avaliação diária a fim de minimizar os riscos do desenvolvimento de infecção( ${ }^{(7)}$.

Diante disso, profissionais de enfermagem que manejam o CVC-TI necessitam de conhecimentos específicos para acompanhar a inserção, manutenção e possíveis complicações relacionadas ao uso dos dispositivos, estes se tornam responsáveis pelo sucesso da manutenção do procedimento, se conscientizando das consequências previsíveis e imputáveis à sua própria ação e omissão, diante do cuidado oferecido ${ }^{(7,8)}$.

Apesar de simples, essas ações exigem da equipe de enfermagem a adoção de medidas com o intuito de evitar que os microorganismos relacionados ao manuseio do cateter ou da própria microbiota do paciente tornem o cateter inutilizado, ou provoquem o adiamento ou interrupção do tratamento devido à infecção( ${ }^{(8)}$. As evidências das contribuições da enfermagem mostram que a punção segura, a manutenção e troca de curativo e a manutenção do sistema fechado são cuidados imprescindíveis para a prevenção de infecção do CVC-TI(6).

Com a constante evolução, as ciências da saúde exigem do enfermeiro permanente atualização e, muitas vezes, especialização, que deve ser adquirida após a formação básica ${ }^{(8)}$. Dessa forma, o presente estuda visa analisar os saberes sobre manuseio de cateter venoso central totalmente implantado de graduandos em enfermagem da Universidade Federal de Sergipe (UFS), ao considerar a relevância desta temática para a atuação profissional e a complexidade do procedimento que exige do enfermeiro habilidades e conhecimentos específicos.

\section{MÉTODO}

Trata-se de pesquisa quantitativa, exploratória, descritiva, com intervenção e análise quantitativa dos dados, realizado entre janeiro e dezembro de 2018.

Fizeram parte da pesquisa graduandos do curso de Enfermagem da Universidade Federal de Sergipe, inseridos no campus Prof. Antônio Garcia Filho, localizado na cidade de Lagarto, Sergipe, Brasil.

Para calcular a amostra foi utilizada a fórmula de Barbetta ${ }^{(9)}$, onde considerou-se uma população de 154 discentes a partir do segundo ciclo de enfermagem, de forma a limitar o erro amostral de $5 \%$, com nível de confiança de 95\%, chegando ao total de III discentes para a amostra.

Foram realizadas durante período descrito, minicurso intitulado "Cateter Venoso Central totalmente implantado (CVC-TI): procedimentos e cuidados".

Os discentes de graduação em enfermagem da UFS foram convidados para o mini curso, que tinha duração de 3 horas, onde foram aplicados os questionários para a coleta de dados. Este continha perguntas relacionadas à indicação e finalidade do CVC-TI; técnica de punção; manutenção do cateter e manuseio do cateter e foram aplicados em duas etapas, antes e após o mini curso.

O questionário foi validado pelo método face validity ${ }^{(10)}$ que consiste na elaboração de um roteiro baseado nos objetivos elencados para o estudo, seguindo os seguintes passos: consenso da primeira versão entre o autor e orientador, adequação da linguagem à população estudada. Convém referir que foi aplicado o roteiro a um número correspondente a $10 \%$ da amostra da pesquisa, o que equivale a I l participantes, antes do início da coleta de dados efetiva, e, estas pessoas foram excluídas da amostra final.

Os mini cursos foram realizados em datas e horários agendados com os participantes da amostra, realizados em ambiente reservado, nas dependências da UFS. Foi garantido o anonimato dos mesmos, bem como a garantia da possibilidade de desistência de participação na 
pesquisa a qualquer momento e o acesso as informações e resultados do estudo.

O estudo foi aprovado pelo Comitê de Ética em Pesquisa da Universidade Federal de Sergipe (UFS), $n^{\circ} \mathrm{CAE}$ 69489917.2 .0000 .5546 , de 05 de junho de 2017, em conformidade com as Resoluções $n^{\circ} 466 / 12$ e 510/16 do Conselho Nacional de Saúde.

Para a tabulação dos dados, foi utilizado o programa da Microsoft Office Excel, e para evitar erros, utilizou-se a ferramenta de validação de dados, disponível no programa utilizado, a qual não permite que sejam inseridas informações que não se enquadram nas alternativas. Os dados foram apresentados de forma numérica e em porcentagem, além de serem mostrados em tabelas e confrontados com a literatura atual.

\section{RESULTADOS}

Participaram da pesquisa, I I I discentes, do segundo ao quinto ciclo de graduação em enfermagem da Universidade Federal de Sergipe, campus Prof Antônio Garcia
Filho. As principais características sociodemográficas estão apresentadas a seguir, na tabela I.

Houve predomínio de discentes do sexo feminino, com idade entre 17 a 22 anos (67,5\%), do segundo ciclo de graduação em enfermagem. Quando questionado sobre o estudo e conhecimento sobre CVC-TI durante a graduação, $87,3 \%$ relataram não ter tido contato e/ou conhecimento sobre a temática. Em relação aos conhecimentos dos discentes antes e após o mini curso, estão descritos nas tabelas 2 a 4 .

Dos III acadêmicos incluídos, 30,6\% dos discentes responderam corretamente aos questionamentos sobre CVC-TI antes do mini curso ofertado, com maior acerto no item manuseio do CVC-TI.

Após o mini curso, 80,5\% responderam corretamente sobre o tema, com melhora significativa dos conhecimentos adquiridos.

maior número de acertos antes do mini curso foi descrito pelos acadêmicos do quinto ciclo e após o mini curso, houve destaque do terceiro ciclo de graduação.

TABELA 1 - Caracterização sociodemográfica dos graduandos em enfermagem da UFS. Lagarto, SE, Brasil, 2014.

\begin{tabular}{l|c|c}
\hline \multicolumn{1}{c|}{ Variáveis } & $\mathbf{N}$ & \% \\
\hline Sexo & & 16,2 \\
\hline Masculino & 18 & 83,7 \\
Feminino & 93 & 67,5 \\
\hline Idade & & 21,6 \\
\hline 17 a 22 & 75 & 10,8 \\
23 a 28 & 24 & \\
29 a 31 & 12 & 38,7 \\
\hline Ciclo de graduação & & 31,5 \\
\hline 2 & 43 & 12,6 \\
3 & 35 & 17,1 \\
4
\end{tabular}

UNIVERSIDADE FEDRAL DE SERGIPE

TABELA 2 - Avaliação do conhecimentos sobre CVC-TI entre discentes de enfermagem da UFS, antes e após participação no minicurso em porcentagem. Lagarto, SE, Brasil, 2014.

\begin{tabular}{|c|c|c|c|c|}
\hline \multirow{2}{*}{ Conhecimento sobre CVC-TI } & \multicolumn{2}{|c|}{ Antes do mini curso } & \multicolumn{2}{|c|}{ Após mini curso } \\
\hline & $\mathrm{N}$ & $\%$ & $\mathrm{~N}$ & $\%$ \\
\hline Sabem o que é CVC-TI & 29 & 26,1 & 97 & 87,5 \\
\hline Sabem das indicações do CVC-TI & 18 & 16,2 & 109 & 98,1 \\
\hline Sabem da ténica de punção do CVC-TI & 21 & 18,9 & 84 & 75,6 \\
\hline Sabem da manutenção do CVC-TI & 28 & 25,2 & 105 & 94,5 \\
\hline Sabem do manuseio do CVC-TI & 74 & 66,6 & 52 & 46,8 \\
\hline Média & \multicolumn{2}{|c|}{$30,6 \%$} & \multicolumn{2}{|c|}{$80,5 \%$} \\
\hline
\end{tabular}

UNIVERSIDADE FEDRAL DE SERGIPE 
TABELA 3 - Avaliação do conhecimento sobre CVC-TI conforme ciclo de graduação, antes do minicurso em porcentagem. Lagarto, SE, Brasil, 2014.

\begin{tabular}{c|c|c|c|c|c|c|c}
\hline $\begin{array}{c}\text { Ciclo } \\
\text { de } \\
\text { graduação }\end{array}$ & $\begin{array}{c}\text { Quantidade } \\
\text { de } \\
\text { alunos }\end{array}$ & $\begin{array}{c}\text { Sabem o que } \\
\text { é CVC-TI }\end{array}$ & $\begin{array}{c}\text { Sabem das } \\
\text { indicações do } \\
\text { CVC-TI }\end{array}$ & $\begin{array}{c}\text { Sabem da } \\
\text { ténica de } \\
\text { punção do } \\
\text { CVC-TI }\end{array}$ & $\begin{array}{c}\text { Sabem da } \\
\text { manutenção do } \\
\text { CVC-TI }\end{array}$ & $\begin{array}{c}\text { Sabem do } \\
\text { manuseio do } \\
\text { CVC-TI }\end{array}$ & $\begin{array}{c}\text { Média de } \\
\text { acertos }\end{array}$ \\
\hline 2 & 38,7 & 65,9 & 72,7 & 36,3 & 59 & 61,3 & 59 \\
\hline 3 & 31,5 & 91,4 & 65,7 & 14,2 & 71,4 & 65,7 & 61,6 \\
\hline 4 & 12,6 & 64,2 & 64,2 & 35,7 & 64,2 & 57,1 & 57 \\
\hline 5 & 17,1 & 57,8 & 73,6 & 21 & 89,4 & 84,2 & 65,2 \\
\hline
\end{tabular}

UNIVERSIDADE FEDRAL DE SERGIPE

TABELA 4 - Avaliação do conhecimento sobre CVC-TI conforme ciclo de graduação, após o minicurso em porcentagem. Lagarto, SE, Brasil, 2014.

\begin{tabular}{c|c|c|c|c|c|c|c}
\hline $\begin{array}{c}\text { Ciclo } \\
\text { de graduação }\end{array}$ & $\begin{array}{c}\text { Quantidade de } \\
\text { alunos }\end{array}$ & $\begin{array}{c}\text { Sabem o } \\
\text { que é CVC- } \\
\text { TI }\end{array}$ & $\begin{array}{c}\text { Sabem das } \\
\text { indicações do } \\
\text { CVC-TI }\end{array}$ & $\begin{array}{c}\text { Sabem da ténica } \\
\text { de punção do } \\
\text { CVC-TI }\end{array}$ & $\begin{array}{c}\text { Sabem da } \\
\text { manutenção do } \\
\text { CVC-TI }\end{array}$ & $\begin{array}{c}\text { Sabem do } \\
\text { manuseio do } \\
\text { CVC-TI }\end{array}$ & $\begin{array}{c}\text { Média de } \\
\text { acertos }\end{array}$ \\
\hline 2 & 38,7 & 84,1 & 97,7 & 81,8 & 93,1 & 34 & 78,1 \\
\hline 3 & 31,5 & 88,5 & 100 & 80 & 91,4 & 60 & 83,9 \\
\hline 4 & 12,6 & 92,8 & 93,8 & 78,5 & 100 & 42,8 & 91,5 \\
\hline 5 & 17,1 & 84,2 & 94,7 & 52,6 & 94,7 & 52,6 & 75,7 \\
\hline
\end{tabular}

UNIVERSIDADE FEDRAL DE SERGIPE

\section{DISCUSSÃO}

O processo de formação acadêmica assume papel fundamental no contexto nacional, tendo em vista as mudanças que vêm acontecendo no sistema de saúde brasileiro, bem como as mudanças no mercado de trabalho Nesse contexto, a universidade enquanto instituição social e os cursos de graduação da saúde devem formar um profissional capaz de desenvolver habilidades teóricas e práticas para atuar com competência na resolução das necessidades reais de saúde das populações. Dessa forma, torna-se de suma importância existência de treinamentos educacionais básico e, até, avançado para melhor preparação do enfermeiro e prevenção de complicações associadas ao CVC-TI, conforme aparece na literatura ${ }^{(11,12)}$.

A idade entre os participantes deste estudo foi entre 17 e 22 anos (67,5\%), matriculados no segundo ciclo de graduação em enfermagem (38,7\%), constituindo uma população jovem.

A avaliação do conhecimento sobre CVC-TI mostrou desconhecimento pelos graduandos em enfermagem antes do mini curso por $70,4 \%$ da amostra. Houve maior número de acertos quando questionados sobre a definição do CVC-TI e manuseio deste; e a maior dificuldade foram relacionadas às indicações e técnicas de punção do CVC-TI, refletindo carência destes sobre o tema abordado.

Ao comparar o conhecimento de estudantes de bacharelado, graduados e pós-graduados em enfermagem sobre ocologia e os cuidados, complicações e aplicações do cateter central, observa-se que $53,6 \%$ dos enfermeiros em primeira formação conhecem sobre o
CVC. Ainda que seja um assunto abordado durante a formação, a taxa de conhecimento sobre ele é relativamente baixa. A área de atuação também tende a ser relevante quanto a continuação desse conhecimento, pois em torno de $32,5 \%$ dos enfermeiros graduados conhecem os processos do CVC. O valor aumenta para $85 \%$ entre os pós-graduados ${ }^{(12)}$.

O CVC-TI, conhecido como "portocath" é um cateter com diâmetro inferior a $\mathrm{IOFr}$, passível de implantação através de veia periférica ou central, e conectado a um reservatório implantado sobre a fáscia muscular do local escolhido para a confecção da loja, geralmente na região do tórax. O reservatório é fabricado em titânio ou plástico com câmara simples ou dupla, há dispositivos valvulados e não valvulados ${ }^{(4,13)}$.

O CVC-TI são utilizados para tratamentos curtos, em situações emergenciais, unidades de tratamento intensivo e em pacientes pós-cirurgicos; ou longos, para pacientes oncológicos. Além da infusão da quimioterapia, o Cateter Venoso Central serve para hidratação, nutrição por solução parenteral, transfusões de hemoderivados e realização de alguns exames ${ }^{(11,14)}$. $O$ local de inserção e o tipo do cateter dependerão da avalição médica e das indicações de protocolos institucionais ${ }^{(1)}$.

A punção do cateter exige uma técnica asséptica, com as substâncias mais indicadas, visando a redução do risco de infecção e eventos adversos, além de cuidados na pele, para evitar a ocorrência de extravasamento dos quimioterápicos e de obstrução. Considerando a atuação, a equipe de enfermagem são os profissionais repsonsáveis pela manutenção do cateter e pela melhora da qualidade do cuidado, previnindo as complicações possíveis ${ }^{(12)}$.A 
utilização de protocolos assistenciais, embasados em evidências, permite ao enfermeiro pensar clinicamente sobre o cuidado individual necessário ${ }^{(15)}$.

A antissepsia deve ser realizada por meio de movimentos em espiral, de dentro para fora, a partir do centro do port e que devem ser executados por, pelo menos, três vezes antes da inserção da agulha.A agulha deve ser introduzida com a mão dominante num ângulo de noventa graus até o fundo do reservatório tocar a parte inferior do dispositivo ${ }^{(15)}$.

A confirmação do correto posicionamento se dá por meio da realização do teste de aspiração, retirando em média $3 \mathrm{ml}$ em crianças e $5 \mathrm{ml}$ em adultos da solução contida na câmara ou até refluir sangue. Nos casos de ausência do retorno venoso, resistência à infusão e/ou dor à infusão, o profissional deve interrompê-la e avaliar o posicionamento adequado da agulha ${ }^{(15)}$.

Quanto ao ciclo de graduação, antes do mini curso, a maior média de acertos $(65,2 \%)$ foi descrita pelos discentes do quinto ciclo, apresentando maiores dificuldades ( $21 \%$ acertaram as respostas) em relação à técnica da punção do CVC-TI.

Após o mini curso, percebeu-se melhora significativa dos conhecimentos adquiridos, ou seja, 80,5\% responderam o mesmo questionário de forma correta, estando a maior dúvida no item manuseio do CVC-TI, apenas $46,8 \%$ responderam corretamente. Por ser uma técnica específica, a punção do CVC-TI é atribuição do enfermeiro, exige conhecimento teórico e habilidade na técnica estéril(6).

Quanto ao ciclo de graduação, discentes do terceiro ciclo apresentaram melhor desempenho, com média de $83,9 \%$ de acertos, sendo a maior dificuldade $(60 \%)$ em relação ao manuseio do CVC-TI.

Em relação ao manuseio, sempre que o cateter está em uso é indicado que o local de inserção da agulha seja protegido por curativo, que também tem por finalidade estabilizar a agulha ${ }^{(15)}$.

A troca deste curativo deve ser realizada quando o mesmo se encontrar sujo, úmido ou solto; excluindo estes casos, recomenda-se a troca a cada 48 horas ou, ao menos, durante uma vez na semana ${ }^{(16)}$. $O$ enfermeiro determina este período a depepnder do material utilizado para cobertura, indicações do fabricante e da sua avaliação clínica. Entretanto, existem estudos que demonstram o número baixo de profissionais de enfermagem que sabem desta informação(11,16).

A manutenção, realizada pelo profissional de enfermagem devidamente capacitado por curso, envolve cuidados com a rede venosa do sítio de inserção, prevenção de complicções e infecções e limpeza e troca do curativo. A qualificação adequada para a manutenção reduz custos financeiros e o desperdício de recusos humanos ${ }^{(17)}$.
A manutenção por salinização do cateter com $20 \mathrm{~mL}$ de soro fisiológico a $0,9 \%$ é um método que realizado com a técnica de flush, atentando para sinais de obstrução. Por não utilizar anticoagulante, torna-se mais segura para o paciente quanto ao risco de sangramento(15). Contudo, também é apontado na literatura a realização do processo de heparinização, na qual a subtância heparina impede a agregação plaquetária e, consequentemente, a oclusão do acesso realizado ${ }^{(18)}$. Ainda não foi relatado diferenças significativas no uso de um ou outro a respeito da melhora da permeabilidade. Logo, a fim de minimizar riscos, é indicada a aplicação de SF 0,9\%(15).

A solução recomendada para a realização da limpeza do sítio de inserção é a clorexidina alcoólica a $2 \%(17)$.

A padronização de condutas, baseada em evidências, por meio de manuais e protocolos é uma forma de garantir uma prática clínica qualificada, pois permite aos profissionais o esclarecimento de dúvidas e orienta a execução das ações, oferecendo maior segurança a estes durante a realização do procedimento(12,15).

Apesar das evidências e da importância do tema para a área da saúde, poucos estudos encontram-se na literatura. Por meio da análise dos questionários notouse que há uma deficiência de conhecimento sobre o tema, o que pode ser preocupante a longo prazo, pois o papel do enfermeiro em pacientes que faz uso dessa terapia vai além de prover o bem estar físico e psíquico do paciente, requer conhecimento técnico-científico do uso deste dispositivo.

O conjunto desses elementos faz com que o tratamento tenha sucesso, pois uma forma de garantir a permanência do CVC-TI, consiste em orientar o paciente e/ ou familiar para o autocuidado, uma das atribuições do profissional enfermeiro. Além disso, o paciente sentese mais assistido pela equipe, trazendo motivação para que ele siga corretamente o tratamento, aumentando as chances de cura.

Diante da grande procura pelo curso por parte dos discentes da UFS, esta pesquisa despertou o interesse dos acadêmicos na temática abordada, a fim de ampliar os conhecimentos na área da oncologia, o que representa uma valorização à educação e ao aperfeiçoamento dos conhecimentos específicos.

O maior número de acertos nas questões após participação do mini curso sobre CVC-TI, mostrou ampliação dos conhecimentos e interesse pela temática abordada.

\section{CONCLUSÃO}

Conclui-se que o conhecimento dos graduandos sobre CVC-TI era deficitário antes do mini curso, sendo melhorado após o mini curso. Há necessidade premente de capacitação profissional e espera-se que o resultados apresentados possam contribuir para dirimir as dúvidas 
apontadas, propiciando maior segurança no manuseio do cateter e prevenindo o surgimento de complicações, o que poderá garantir assistência de enfermagem qualificada e, consequentemente, melhor qualidade de vida aos pacientes atendidos.

Os resultados apontados mostraram a necessidade de inserção deste tema nos cursos de graduação em enfermagem do campus onde se realizou a pesquisa, com o objetivo de ensinar/atualizar os graduandos em relação às técnicas de manipulação e os principais cuidados aos pacientes com este dispositivo, e assim, prepará-los para atuar nos serviços de saúde. 


\section{REFERÊNCIAS}

I. Instituto Nacional de Câncer José Alencar Gomes da Silva. Estimativa 2020: incidência de câncer no Brasil. Rio de Janeiro: INCA; 2019 [acesso em 21 fev 2020]. Disponível em: https://www.inca.gov.br/sites/ufu.sti.inca.local/files//media/document//estimativa-2020-incidencia-de-cancer-no-brasil.pdf

2. Brasil. Ministério da Saúde. Secretaria de Atenção à Saúde - SAS/MS. Manual de bases técnicas da oncologia - SIA/ SUS - Sistema de Informações Ambulatoriais. 25 ed. Brasília: Ministério da Saúde; 2019.

3. Da Silva FP, Pereira AD. A Enfermagem no Cuidado a Pacientes Oncológicos que Fazem uso de Cateter Venoso Totalmente Implantado. Discip. Sci., Sér. Ciênc. Biol. Saúde. 200I;2(I):4I-52.

4. Zerati AE, Wolosker N, De Luccia N, Puech-Leão P. Cateteres venosos totalmente implantáveis: histórico, técnica de implante e complicações. JVasc Bras 2017; 16(2): I28-I39.

5. Brasil. Ministério da Saúde. Documento de referência para - Programa Nacional de Segurança do Paciente. Brasília: Ministério da Saúde; 2014.

6. Oliveira TF, Rodrigues MCS. Enfermagem Na Prevenção De Infecção Em Cateter Totalmente Implantado No Paciente Oncológico. Cogitare Enferm 2016; 2 I (2): 01-05.

7. Brasil. Lei $n^{\circ} 7.498$, de 25 de junho de 1986. Dispõe sobre a regulamentação do exercício da enfermagem e dá outras providências. Brasília: Diário Oficial da União; 1986. Disponível em: http:///www.planalto.gov.br/ccivil_03/ leis/17498.htm

8. Oliveira DAL, Fontes RA, Da Silva, MB. Cuidados de enfermagem ao paciente oncológico portador de cateter totalmente implantado.VITTALLE-Revista de Ciências da Saúde 20I9; 3I(I): 52-60.

9. Barbetta, PA. Estatísticas aplicadas às Ciências Sociais. I ed. Florianópolis: UFSC, 1994.

10. Bessa, NM.Validade - o conceito, a pesquisa, os problemas de provas geradas pelo computador. Estudos em avaliação educacional [periódico na Internet]. 2007 Mai/Ago [acesso em dez 2018]; I8(37). Disponível em: http://publicacoes.fcc. org.br/ojs/index.php/eae/article/view/2093/2050

II. Abu Sharour L, Subih M, Yehia D, Suleiman K, Salameh AB, Al Kaladeh M. Teaching module for improving oncology nurses' knowledge and self-confidence about central line catheters caring, complications, and application: A pretest-posttest quasi-experimental design. J Vasc Nurs. 2018 Dez;36(4):203-207.

12. Abu Sharour L. Oncology nurses' knowledge about central line catheter: Caring, complications, and applications among cancer patients-A cross-sectional study. J Vasc Nurs. 2018 Set;36(3): I45- I 48.

13. Peixoto RMA, Souza SR, Silva JC, Mendes EMS, Sória DAC, Fontes LM. Complicações do cateter port a cath: subsídios para os cuidados de enfermagem. Revista Enfermagem Atual In Derme. 2019;87(Esp).

14. Mason TM, Ferrall SM, Boyington AR, Reich RR. Central venous access devices: an investigation of oncology nurses' troubleshooting techniques. Clin J Oncol Nurs. 2014 Ago; 18(4):421-5.

15. Fonseca DF, Oliveira PP, Amaral RAC, Nicoli LHS, Silveira EAA, Rodrigues AB. Protocolo de cuidados com cateter venoso totalmente implantado: uma construção coletiva. Texto Contexto - Enferm. 2019;28.

16. Al Qadire M. Oncology nurses' knowledge of guidelines for preventing catheter related bloodstream infections. Am J Infect Control. 2017 set;45(9):e95-e97

17. Homo RFB, Lima AFC. Custo direto da manutenção da permeabilidade de cateter venoso central totalmente implantado. Rev. Latino-Am. Enfermagem [Internet]. 2018 [acessado em I 8 fev 2020];26:e3004. Disponível em: http://www.scielo. br/pdf/rlae/v26/pt_0104-I I69-rlae-26-e3004.pdf

18. Pires NN,Vasques $\mathrm{Cl}$. Conhecimento de enfermeiros acerca do manuseio de cateter totalmente implantado. Texto contexto - enferm. 2014 Jun;23(2):443-450.

Recebido: 2020-06- 15

Aceito: 2020-07-17 